\title{
Evaluation of Extensor Pollicis Brevis as a Recipient of Tendon Transfer for Thumb Extension
}

\author{
Praveen Bhardwaj ${ }^{1}$ Poonacha Puchimada Muddappa ${ }^{1}$ Dadi Bindesh ${ }^{1} \quad$ Shanmuganathan Raja Sabapathy \\ ${ }^{1}$ Departments of Plastic, Hand, and Reconstructive Microsurgery, \\ Address for correspondence Praveen Bhardwaj, MS, DNB \\ (Ortho), FNB (Hand and Microsurgery), EDHS, Department of \\ Plastic, Hand and Reconstructive Microsurgery, Ganga Hospital, \\ 313-Mettupalayam Road, Sai Baba Colony, Coimbatore 641 043, \\ Tamil Nadu, India (e-mail: drpb23@gmail.com).
}

Indian J Plast Surg 2019;52:171-177

\begin{abstract}
Introduction Rerouting of the extensor pollicis longus (EPL) is the standard part of tendon transfer surgery for thumb extension. It is done to overcome the ulnar vector of the EPL action. Extensor pollicis brevis (EPB), however, produces better thumb abduction and extension by virtue of its radial vector. The described anatomical variation of EPB extending the thumb interphalangeal joint (IPJ), therefore, gives the "best combination" of movements by a single-thumb extensor tendon.

Materials and Methods We performed this transfer in six patients in whom the EPB was found to be extending the IPJ while checked intraoperatively. Three of these six patients were cases of radial nerve palsy and the other three presented with brachial plexus palsy. The outcome was assessed by measuring palmar and radial abduction of the thumb, Kapandji's score, and Bincaz's scale.

Results We found satisfactory results in all the six patients. In our series, patients

Keywords

- brachial plexus

- radial nerve palsy

- rerouting

- tendon transfer

- thumb extension

- wrist drop

- wrist extension had an average radial extension of the thumb of 29.2 degrees and an average palmar abduction of the thumb of 65.7 degrees. On evaluation with the Bincaz score; one patient had excellent result, three patients had good results, and two patients had fair results.

Conclusion In situations where EPL rerouting is not possible (as in cases where the donor tendon needs to reach the thumb from the ulnar side, for example, flexor carpi ulnaris), transfer to the EPB, provided it is extending the thumb IPJ, would produce better extension and abduction of the thumb than the transfer to the EPL.
\end{abstract}

\section{Introduction}

Extension of the fingers and the thumb adds to prehension of the hand by increasing its ability to open the hand for grasping larger objects. For restoration of the hand function in radial nerve palsy, the triple-tendon transfer of pronator teres (PT) to extensor carpi radialis brevis (ECRB), flexor carpi ulnaris (FCU)/flexor carpi radialis to extensor digitorum communis (EDC), and palmaris longus (PL) to rerouted extensor pollicis longus (EPL) has been recommended. Our results with this set of triple-tendon transfers are satisfactory as described in the study by Latheef et al. ${ }^{1}$ Rerouting of EPL volarly and transfer to the PL was described by Scuderi ${ }^{2}$
DOI https://doi.org/

10.1055/s-0039-1696617

ISSN 0970-0358. to achieve a combined extension and abduction, describing excellent results. Over the years, this has become the standard transfer for thumb extension. Brand in his study of the clinical mechanics of the hand has concluded that the EPL is an extensor and adductor at the carpometacarpal (CMC) joint and the extensor pollicis brevis (EPB) is an extensor and abductor. ${ }^{3}$ This is because the EPL is ulnar to the axis of the CMC joint and the EPB is radial to the CMC joint axis ( - Fig. 1). Therefore, it is necessary to reroute EPL when it is used as a recipient for thumb extension transfer (-Fig. 2). The EPB, on the other hand, produces good opening of the first web by abduction at the CMC joint by virtue of being radial to the CMC joint axis. However, the 


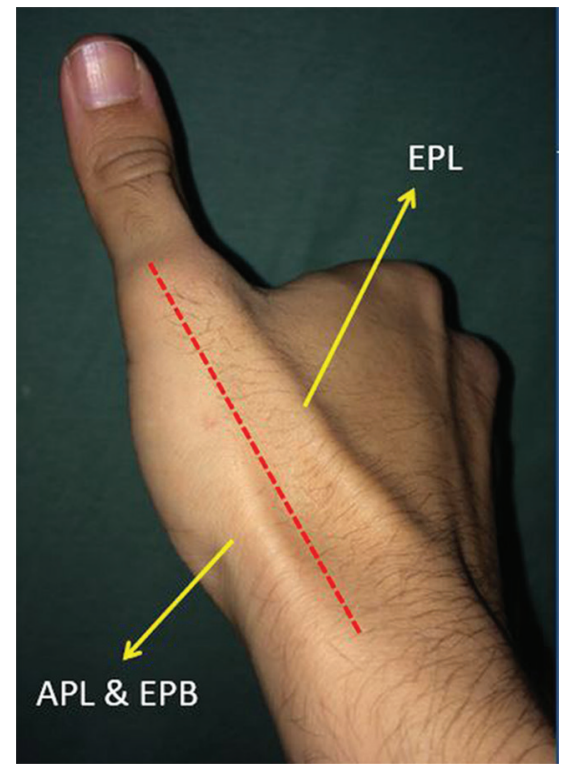

Fig. 1 A clinical picture showing the surface marking of the extensor pollicis longus and the extensor pollicis brevis with respect to the metacarpophalangeal and carpometacarpal joints. The extensor pollicis longus being ulnar to the axis acts as an extensor and adductor while the extensor pollicis brevis acts as an extensor and abductor (refer text). APL, abductor pollicis longus; EPB, extensor pollicis brevis; EPL, extensor pollicis longus.

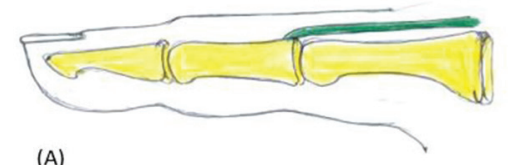

(A)

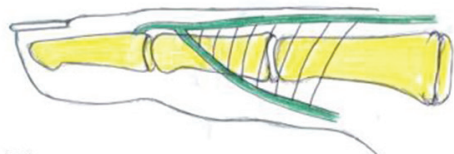

(B)

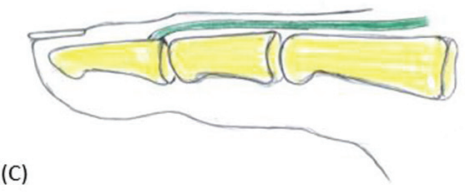

Fig. 3 The representative diagram showing the anatomical variations in the insertion patterns of the extensor pollicis brevis. (A) The standard distal attachment of the extensor pollicis brevis on to the dorsal base of the proximal phalanx; (B) a distal attachment to the extensor hood; and (C) a distal attachment of a distinct extensor pollicis brevis tendon onto the dorsal base of the distal phalanx (Alemohammad et $\mathrm{al}^{10}$ ).

EPB does not usually extend the interphalangeal (IP) joint. The described anatomical variation of the EPB extending the thumb IP joint (-Fig. 3), therefore, gives the "best combination" of movements for a single-thumb extensor tendon, that is, abduction at the CMC joint, extension at the metacarpophalangeal joint (MCPJ), and extension at the IP joint. We hypothesized that in such patients (having

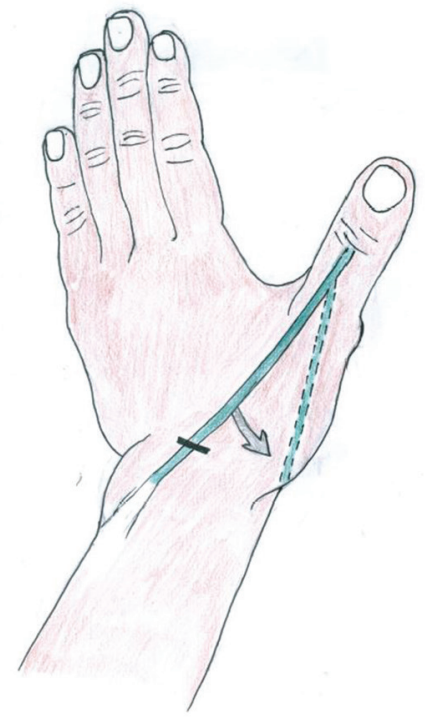

Fig. 2 Line diagram showing the concept of rerouting of the extensor pollicis longus, the extensor pollicis longus tendon is removed from its normal location (around the Lister's tubercle) and transferred to more radial direction (arrow) so that instead of working as an extensor and adductor it can start working as an abductor and extensor at the thumb joints.

an anatomical variation of EPB which is extending the IP joint of thumb) tendon transfer to the EPB provides better results than a rerouted EPL transfer. Our study aimed to evaluate the efficacy of such tendon transfers to the EPB.

In a patient with brachial plexus palsy, we chose FCU as a favorable donor for EDC and EPL based on the motor profile. We planned to attach FCU to both EDC and EPL (split FCU transfer). During intraoperative traction on the EPL, we noted an adduction movement which could not be addressed by rerouting, as the donor tendon was reaching the EPL from the ulnar side ( - Fig. $4 A$ ). Since the rerouting was not possible, we inspected the EPB. On applying traction on the EPB, we noted good radial abduction and extension of the thumb ( - Fig. 4B). FCU was attached to EPB to get thumb extension. Relying on this outcome of abduction and extension in this patient, we have performed this transfer in six patients.

\section{Materials and Methods}

This study is a retrospective analysis of six patients who underwent tendon transfer for wrist and digital drop. Among these six patients, three patients had radial nerve palsy and three patients had brachial plexus palsy. In these patients, transfer of the donor tendon to the rerouted EPL was planned for the thumb extension. When EPB was evaluated intraoperatively, it was found to extend the IP joint also (-Fig. 4B). Therefore, in these patients, EPB was used as the recipient instead of a rerouted EPL. In patients with radial nerve palsy after the PT to ECRB and FCU to EDC transfers were completed, the PL was delivered into the dorsal forearm wound. With the thumb held in extended and abducted position, the PL was weaved through EPB. In patients with brachial plexus palsy, the PT to ECRB was done 

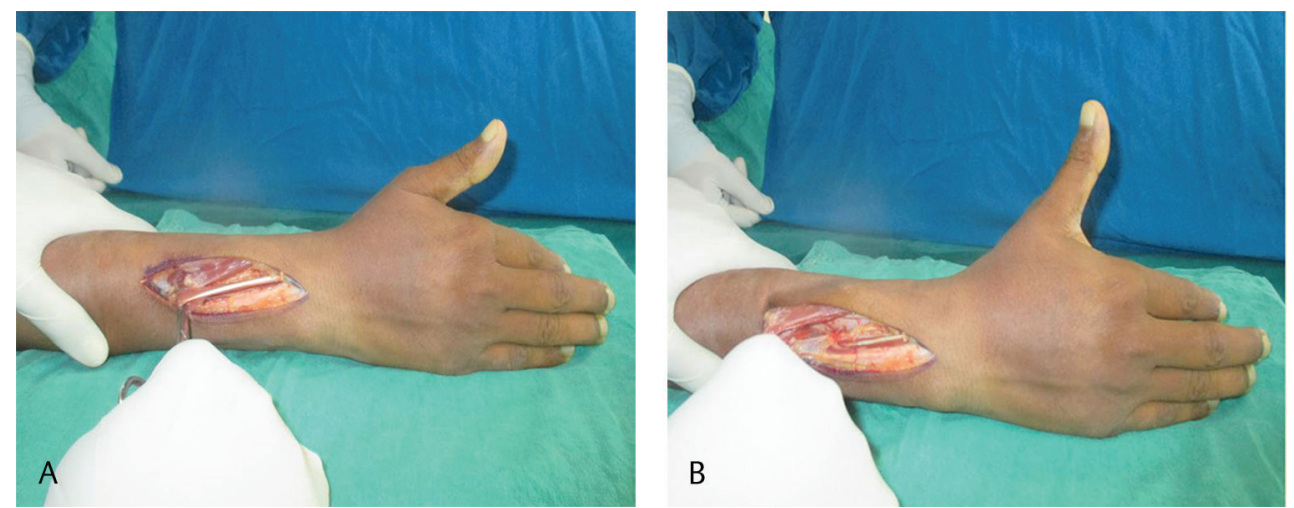

Fig. 4 Intraoperative photograph showing the movement of the thumb on applying traction to extensor pollicis longus (A) and extensor pollicis brevis tendon (B). One can note the thumb extension and adduction produced by traction on extensor pollicis longus (A) and abduction and extension produced on applying traction to extensor pollicis brevis (B).
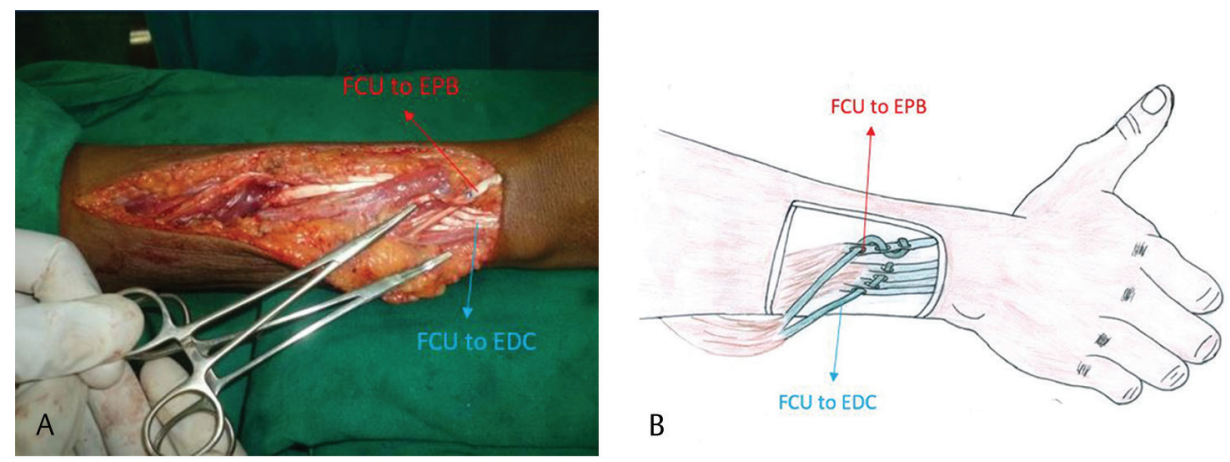

Fig. 5 (A) Intraoperative photograph and (B) the line diagram showing the direction of split flexor carpi ulnaris transfer (hemostat showing the direction) to reach the extensor pollicis brevis and the extensor digitorum communis slips for insertion. EDC, extensor digitorum communis; EPB, extensor pollicis brevis; FCU, flexor carpi ulnaris.

Table 1 Bincaz score for evaluation of the results of tendon transfer for wrist and digital extension

\begin{tabular}{|l|l|l|l|l|}
\hline Points & 3 & 2 & 1 & 0 \\
\hline Wrist extension & & $>29$ degrees & $0-29$ degrees & 0 \\
\hline MCP joint extension & Full & $\begin{array}{l}\text { Loss of extension }<100 \\
\text { degrees }\end{array}$ & Loss of extension $>100$ degrees \\
\hline $\begin{array}{l}\text { First web space } \\
\text { opening }\end{array}$ & $>39$ degrees & $30-39$ degrees & $<30$ degrees \\
\hline Patient satisfaction & Excellent & Good & Fair & Poor \\
\hline
\end{tabular}

Abbreviation: MCP, metacarpophalangeal.

in one patient who had a strong PT and in other two patients with paralyzed PT, we used flexor digitorum superficialis (FDS) of middle finger to the ECRB for wrist extension. FCU, which was strong in all these three patients, was transferred to EDC for finger extension (split FCU into two cases). For the thumb extension, in one patient, FDS of ring finger was transferred to EPB and other two cases, one slip of split FCU was transferred to the EPB and another to the EDC ( - Figs. 5A and $\mathbf{B}$ ). Postoperative mobilization was started after 4 weeks. Patients were allowed to use their hand at 2-month post surgery.

We evaluated these patients at a minimum of 6-month follow-up (range: 6 months-2 years) for range of motion of the thumb, pinch strength, and outcomes by the Bincaz score
(-Table 1). We measured the radial extension of the thumb at the $\mathrm{CMC}$ joint, in the plane of the palm, as the angle between the line along radial subcutaneous border of the first metacarpal and the index finger ray ( - Fig. $\mathbf{6 A}$ ). This was measured with the IP joint in extension, as a flexed IP joint reduces the radial extension of the thumb. The palmar abduction was measured at the CMC joint in the plane perpendicular to the flat palm, as the angle between the dorsal subcutaneous border of the proximal phalanx of the thumb and the palmar border of the index ray (-Fig. 6B). The Kapandji score was measured to assess any restriction in opposition of the thumb. The strengths of pulp pinch and key pinch were measured using a pinch meter. 

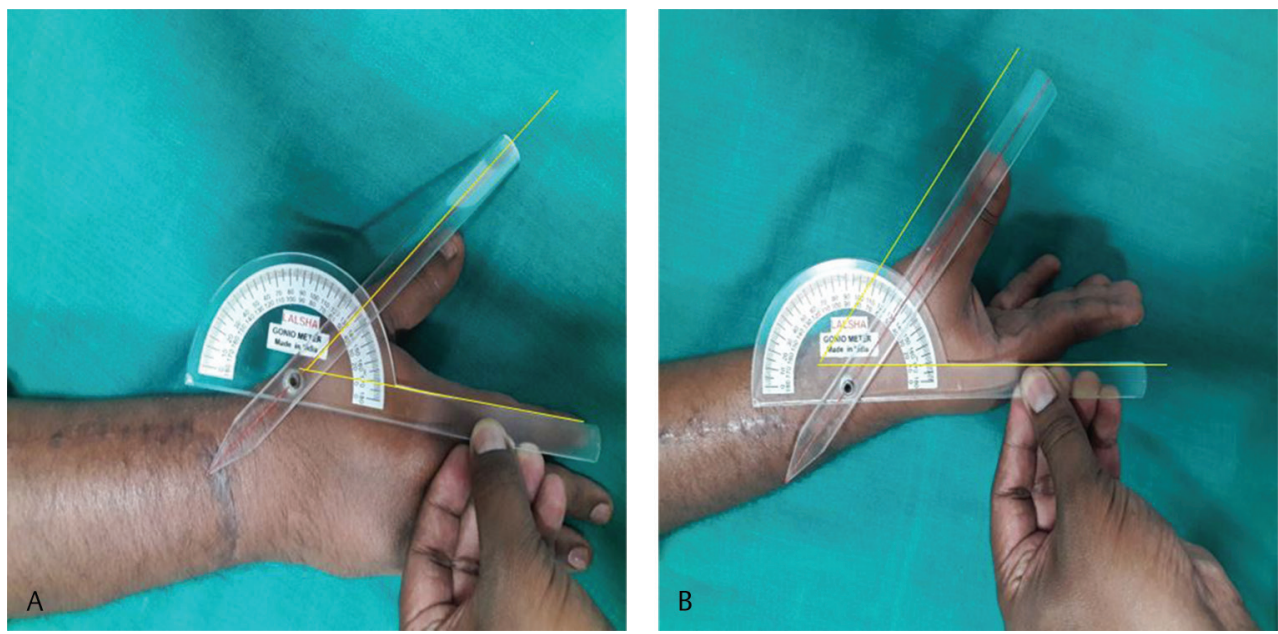

Fig. 6 (A) Clinical picture showing the measurement of the radial abduction and (B) the palmar abduction achieved after the tendon transfer procedure.

Table 2 Patient details and the result achieved in our series

\begin{tabular}{|c|c|c|c|c|c|c|c|c|}
\hline $\begin{array}{l}\text { Serial } \\
\text { number }\end{array}$ & Diagnosis & $\begin{array}{l}\text { Type of } \\
\text { transfer }\end{array}$ & $\begin{array}{l}\text { Radial } \\
\text { abduction } \\
\text { (degree) }\end{array}$ & $\begin{array}{l}\text { Palmar } \\
\text { abduction } \\
\text { (degree) }\end{array}$ & $\begin{array}{l}\text { IPJ flexion } \\
\text { (degree) }\end{array}$ & $\begin{array}{l}\text { Key } \\
\text { pinch }\end{array}$ & Pulp pinch & $\begin{array}{l}\text { Kapandji's } \\
\text { score }\end{array}$ \\
\hline 1 & $\begin{array}{l}\text { Brachial } \\
\text { plexus } \\
\text { palsy } \\
(\mathrm{C} 5-\mathrm{C} 7)\end{array}$ & FDS-EPB & 30 & 60 & 60 & 5.0 & 3.0 & 10 \\
\hline 2 & $\begin{array}{l}\text { Radial } \\
\text { nerve } \\
\text { palsy }\end{array}$ & PL-EPB & 25 & 66 & 90 & 4.5 & 3.5 & 10 \\
\hline 3 & $\begin{array}{l}\text { Radial } \\
\text { nerve } \\
\text { palsy }\end{array}$ & PL-EPB & 30 & 70 & 68 & 4.5 & 3.0 & 10 \\
\hline 4 & $\begin{array}{l}\text { Brachial } \\
\text { plexus } \\
\text { palsy } \\
(\mathrm{C} 5-\mathrm{C} 7)\end{array}$ & FCU-EPB & 35 & 68 & 70 & 5.0 & 3.6 & 10 \\
\hline 5 & $\begin{array}{l}\text { Radial } \\
\text { nerve } \\
\text { palsy }\end{array}$ & PL-EPB & 35 & 60 & 80 & 5.2 & 4.0 & 10 \\
\hline 6 & $\begin{array}{l}\text { Brachial } \\
\text { plexus } \\
\text { palsy } \\
(\mathrm{C} 5-7)\end{array}$ & FCU-EPB & 20 & 70 & 85 & 5.3 & 3.0 & 10 \\
\hline \multicolumn{3}{|l|}{ Mean } & 29.2 & 65.7 & 75.5 & 4.9 & 3.4 & 10 \\
\hline
\end{tabular}

Abbreviations: EPB, extensor pollicis brevis; FCU, flexor carpi ulnaris; FDS, flexor digitorum superficialis; IPJ, interphalangeal joint; PL, palmaris longus.

\section{Results}

At an average follow-up of 9 months (range: 6 months2 years), patients had average radial extension of the thumb of 29.2 degrees and an average palmar abduction of 65.7 degrees. The mean key pinch was $4.9 \mathrm{~kg}$ and pulp pinch was $3.4 \mathrm{~kg}$. All patients had a Kapandji score of 10 (-Table 2). On evaluation with the Bincaz score, one patient had an excellent result, three patients had good results, and two patients had fair results ( - Fig. 7 ).

\section{Discussion}

In radial nerve palsy, there is loss of extension of the wrist, fingers, and thumb. These patients have difficulty in bringing the thumb out of the palm. The traditional recipients have been ECRB, EDC, and EPL. The transfer of PL to rerouted EPL has been performed routinely since its description by Scuderi. ${ }^{2}$

The thumb is brought out of the palm by three tendons, all supplied by radial nerve, which act on the three joints. 

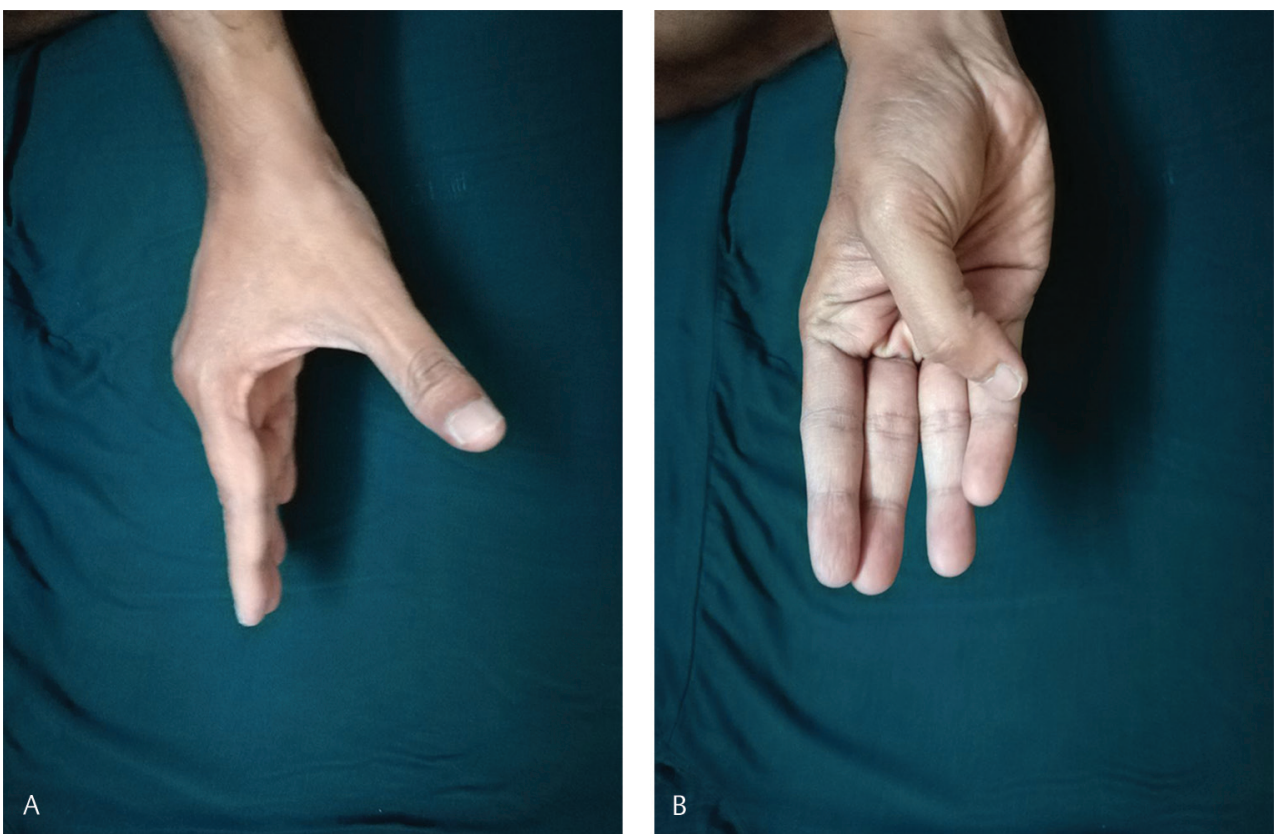

Fig. 7 Clinical pictures showing good long-term results of the tendon transfer to extensor pollicis brevis for thumb extension. (A) Shows good palmar abduction achieved and (B) shows comfortable full opposition, indicating the suitable tension in the transfer and restoration of good function.

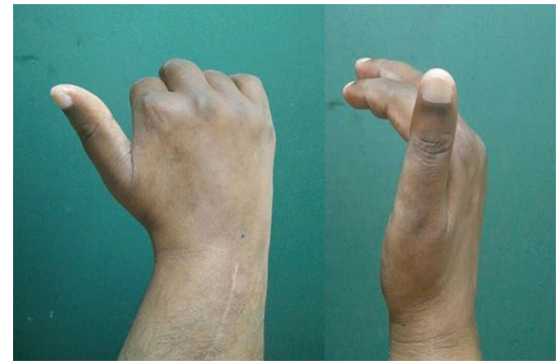

Fig. 8 Clinical picture of a patient with brachial plexus palsy who underwent flexor carpi ulnaris transfer to extensor digitorum and extensor pollicis longus for finger and thumb extension, respectively. Due to the paucity of donors, only flexor carpi ulnaris was available and hence, it was used for both finger and thumb extension. Since the flexor carpi ulnaris reaches dorsum from the ulnar side rerouting of the extensor pollicis longus was not possible. The thumb extension achieved in this patient was not good because the thumb had as adduction moment while extension.

The abductor pollicis longus (APL) brings about movement of the CMC joint, EPB primarily at the MCPJ and the EPL primarily at the IP joint, and also MCPJ and CMC joint as it crosses these joints. EPL is chosen as a recipient for tendon transfer in radial nerve palsy, since it traverses all the three joints. APL and EPB are radial to the MCP and CMC joint axis, and they produce radial extension. Whereas, EPL is ulnar to the axis of the thumb CMC joint; therefore, it produces adduction movement of the thumb. Due to its ulnar vector across the CMC and MCPJs, it has to be rerouted to act as an extensor and abductor. Rerouting is a standard part of radial nerve tendon transfer since its description by Scuderi, ${ }^{2}$ but it requires donor tendon to approach the thumb from the radial side. If split FCU or FCU along with EDC is used as donor, then rerouting is technically not possible, as these tendons would reach the thumb from the ulnar side. In such patients, the results are poor as the thumb goes into extension and adduction rather than abduction ( - Fig. 8). Variations in the insertion of EPB, into the extensor hood or base of the distal phalanx, have been described, leading the EPB to extend the IP joint also (- Fig. 3). Since it is radial to the CMC and MCPJ axis, it should act as a better radial extensor (abductor).

The standard anatomical description of the EPB, found in the current anatomical textbooks, describes its origin as the posterior surface of the radius and adjacent part of the interosseous membrane, distal to the origin of the abductor APL. Its tendon is described to insert into the posterior surface of the base of the proximal phalanx of the thumb after passing under the extensor retinaculum. ${ }^{4}$ The primary function of EPB has been described to cause extension of the MCPJ of the thumb, with contributions to abduction of the thumb and carpus, as well as stabilizing the MCPJ of the thumb, by integrating into the extensor hood. 5,6

Dawson and Barton, ${ }^{7}$ from their study of the EPB on 16 cadaveric hands, concluded that this standard description does not reflect reality and that EPB tendon anatomy varies considerably from the standard description, even between two hands of the same individual. The EPB and APL are considered to differentiate from a common muscle mass and are found as completely separate entities only in humans and gibbons. ${ }^{7,8}$ This theory of a common origin for the EPB and APL is further supported by the fact that, in certain cases, the EPB muscle is fused to a variable extent with the muscle belly of the APL as documented by Kulshreshtha et al. ${ }^{6}$ They also found the absence of a separate muscle belly in three hands (6.8\%) of a total of 44 cadaveric hands. They further describe the variations of insertion of the EPB. They found that in $25 \%$ of the cases, the tendon was inserted wholly into the base of proximal phalanx; in $2 \%$ of cases, the tendon was inserted exclusively into the extensor hood, in $25 \%$ tendons 
were inserted partly into the base of the proximal phalanx and partly into the extensor hood. Also, 27\% tendons showed all of these attachments, that is, partial attachment to the base of the proximal phalanx and to the extensor hood, coupled with prolongation to the distal phalanx. Twenty percent tendons were attached only to the extensor hood, whereas they were prolonged into a combined insertion with the tendon of EPL into the distal phalanx. In these studies, there is no clear description whether these variable insertions caused extension of the IP joint or not. Brunelli and Brunelli, ${ }^{5}$ in their study of the EPB in 52 cadaveric hands, reported that the tendon inserted wholly into the extensor hood, in $69.2 \%$ of cases, insertion of the tendon partially to the base of the proximal phalanx and extensor hood in $19.2 \%$ and base of the distal phalanx direct insertions in four cases (7.5\%).

In their study of anatomical variations in the first extensor compartment of the wrist, Jackson et $\mathrm{al}^{9}$ found 57 (19\%) cases where the EPB had dual insertion with one slip into the proximal phalanx and the other inserting to the EPL. They also found an additional 16 (5.3\%) cases where traction on the EPB tendon caused IP joint extension, but the dual insertion was not demonstrated or the dissection was not completed. Alemohammad et $\mathrm{al}^{10}$ in their study of 90 cadaveric wrists and 143 patients with De Quervain's tenosynovitis, found $21.1 \%$ of EPB in the cadavers to cause extension of the IP joint, and $39.2 \%$ of the patients with De Quervain's tenosynovitis to have EPB causing extension of the IP joint.

Hence, these studies suggest that the incidence of the EPB extending the IP joint also should be upward of $20 \%$ of the population. In our institute, on serially examining the effect of EPB on the IP joint in 46 consecutive cases undergoing tendon transfer in the region, we noted that in 16 patients (34.8\%), the EPB was extending the IP joint also. Hence, a similar proportion of radial nerve/brachial plexus palsy patients (one third) is potential candidates for an EPB, in lieu of a rerouted EPL transfer.

Rerouting the EPL may not actually be possible for all patients. When the FCU is used for both finger and thumb extension, the donor tendon comes from the ulnar aspect of the thumb precluding volar radial shift of the EPL. Such a transfer may be necessary in the absence of the PL or due to paucity of donors in brachial plexus palsy. Single-FCU tendon transfers have also been described for high-radial nerve palsies and no rerouting is possible if they are chosen. ${ }^{11,12}$ In addition, the subcutaneous rerouting of the EPL tendon causes a bowstringing on the volar aspect of the wrist which may not be cosmetically acceptable.

Ropars et $\mathrm{al}^{13}$ in their study of radial nerve tendon transfer in 15 patients, reported mean abduction of the thumb as 54 degrees (range: 0-70 degrees) and opposition as 8.7 (range: 3-10) points on Kapandji's scale. When FCU was used as a single donor for both fingers and thumb extension, Gousheh and Arasteh ${ }^{11}$ reported thumb abduction of 38 degrees (range: 35-42 degrees) and Al-Qattan ${ }^{14}$ reported thumb abduction of 55 degrees (range: 50-60 degrees) achieved in their series. In our study, the mean palmar abduction was 65.7 degrees. The articles in the literature do not specifically mention about radial abduction. However, our patients had a radial abduction of 27.5 degrees. Altintas et a ${ }^{15}$ reported the mean abduction of the thumb in their series as 46 degrees. Using Bincaz score, Ropars et $\mathrm{al}^{13}$ in their study of 15 patients found that 11 had excellent, two good, one fair, and one bad result. Al-Qattan ${ }^{14}$ reported the overall result as per the Bincaz scale was excellent in 12 patients and good in the remaining three patients. In the present study, on evaluation with the Bincaz score, one patient had an excellent result, three patients had good results, and two patients had fair results ( - Figs. $7 \mathbf{A}$ and $\mathbf{B}$ ). Both the patients with fair results were the cases of brachial plexus palsy with limited donor options and overall upper limb weakness where the patients reported their overall satisfaction of function as fair contributing to the "fair outcome" on Bincaz's scale with score of 5 (-Table 1), but the improvement in thumb extension was good in them as indicated by the range of thumb movements achieved in our series ( $\mathbf{- T a b l e ~} \mathbf{2}$ ).

Transfers to the EPB have been used earlier in spastic hands with thumb in palm deformity patients who have weakness of the thumb extensors. Transfer to the EPB has been preferred for a similar reason that it brings about thumb abduction and extension. Transfers to the EPL may not correct thumb in palm deformity because of its adductor moment to the thumb. ${ }^{16,17}$

Jackson et al, ${ }^{9}$ in their study of the EPB variations, have speculated on a transfer to the EPB in radial nerve palsy. They suggested traction on the EPB to check whether it extends the IP joint before deciding on the final transfer. However, no such case reports and results have since appeared in the literature. We followed a similar methodology to find good results with use of the EPB as a recipient for thumb extension transfer when it extends the IP joint as well. In patients with this favorable anatomical variation (34.8\%), the EPB insertion provides an acceptable alternative to rerouting the EPL. In ulnar-sided donor transfers, such a transfer to the EPB is preferable to a transfer to the "unrerouted" EPL. We recommend that the EPB action is evaluated in all cases of thumb extension tendon transfer when rerouting is not possible; if found extending the IP joint also, the EPB is a better recipient for the transfer for thumb extension.

There are few shortcomings of using the EPB as follows:

- EPB may be very thin and muscular, not allowing good tendon to tendon opposition. In such cases, it is better to transfer to EPL. We encountered this situation in two cases.

- Decision for transfer to EPB can be taken only after exploration.

- If one does not find the favorable EPB anatomy and the available donor tendon is reaching the thumb from the ulnar side which precludes rerouting, one could use the technique described by Colantoni Woodside and Bindra ${ }^{18}$ in which the EPL is divided proximally, withdrawn distally through a small incision over the metacarpal, and then tunneled back through the first compartment in a retrograde manner to get it in line with the EPB. It could then be sutured to the donor tendon.

- Alternatively, one could divide the EPL from its distal insertion on the ulnar side of the extensor apparatus, withdraw it proximally, and reroute it through the first compartment 
and suture it on the radial side of the extensor apparatus at the MCPJ to get the same effect.

Based on our observations from this study, we recommend to use EPB as a recipient for the tendon transfers in which the donor tendon reaches the thumb from the ulnar side provided EPB is found to extend the IP joint. In other cases, when the possibility of EPL rerouting is present, the results achieved by transfer to EPB in this series are comparable to the rerouted EPL transfer.

\section{Conclusion}

Extensor pollicis brevis (EPB) produces better thumb abduction and extension by virtue of its radial vector as compared with the EPL which has a more ulnar vector necessitating rerouting. The described anatomical variation of EPB extending the thumb interphalangeal joint, therefore, gives the "best combination" of movements by a single-thumb extensor tendon. Hence, in situations where EPL rerouting is not possible (as in cases where the donor tendon needs to reach the thumb from the ulnar side, for example, split flexor carpi ulnaris) transfer to the EPB, provided it is extending the thumb IPJ, would produce better extension and abduction of the thumb than the transfer to the 'un-rerouted' EPL.

\section{Conflicts of Interest}

There are no conflicts of interest to declare.

\section{References}

1 Latheef L, Bhardwaj P, Sankaran A, Sabapathy SR. An objective functional evaluation of the flexor carpi ulnaris set of triple tendon transfer in radial nerve palsy. J Hand Surg Eur Vol 2017;42(2):170-175

2 Scuderi C. Tendon transplants for irreparable radial nerve paralysis. Surg Gynecol Obstet 1949;88(5):643-651

3 Brand PW, Hollister AM, Clinical Mechanics of the Hand. 3rd ed. St. Louis, MO: Mosby; 1999;384

4 Snell RS, The upper limb. In: Clinical Anatomy by Regions. 9th ed. Philadelphia, PA: Lippincott Williams \& Wilkins; 2012;334-433
5 Brunelli GA, Brunelli GR. Anatomy of the extensor pollicis brevis muscle. J Hand Surg [Br] 1992;17(3):267-269

6 Kulshreshtha R, Patel S, Arya AP, Hall S, Compson JP. Variations of the extensor pollicis brevis tendon and its insertion: a study of 44 cadaveric hands. J Hand Surg Eur Vol 2007;32(5):550-553

7 Dawson S, Barton N. Anatomical variations of the extensor pollicis brevis. J Hand Surg [Br] 1986;11(3):378-381

8 Willan PL, Humpherson JR. Concepts of variation and normality in morphology: important issues at risk of neglect in modern undergraduate medical courses. Clin Anat 1999;12(3):186-190

9 Jackson WT, Viegas SF, Coon TM, Stimpson KD, Frogameni AD, Simpson JM. Anatomical variations in the first extensor compartment of the wrist. A clinical and anatomical study. J Bone Joint Surg Am 1986;68(6):923-926

10 Alemohammad AM, Yazaki N, Morris RP, Buford WL, Viegas SF. Thumb interphalangeal joint extension by the extensor pollicis brevis: association with a subcompartment and de Quervain's disease. J Hand Surg Am 2009;34(4):719-723

11 Gousheh J, Arasteh E. Transfer of a single flexor carpi ulnaris tendon for treatment of radial nerve palsy. J Hand Surg $[\mathrm{Br}]$ 2006;31(5):542-546

12 Sankaran A, Thora A, Arora S, Dhal A. Single tendon transfer of the flexor carpi ulnaris for high radial nerve injury. J Orthop Surg (Hong Kong) 2015;23(3):345-348

13 Ropars M, Dréano T, Siret P, Belot N, Langlais F. Long-term results of tendon transfers in radial and posterior interosseous nerve paralysis. J Hand Surg [Br] 2006;31(5):502-506

14 Al-Qattan MM. Tendon transfer for radial nerve palsy: a single tendon to restore finger extension as well as thumb extension/ radial abduction. J Hand Surg Eur Vol 2012;37(9):855-862

15 Altintas AA, Altintas MA, Gazyakan E, Gohla T, Germann G, Sauerbier M. Long-term results and the Disabilities of the Arm, Shoulder, and Hand score analysis after modified Brooks and D’Aubigne tendon transfer for radial nerve palsy. J Hand Surg Am 2009;34(3):474-478

16 Tonkin MA, Hatrick NC, Eckersley JR, Couzens G. Surgery for cerebral palsy part 3: classification and operative procedures for thumb deformity. J Hand Surg [Br] 2001;26(5):465-470

17 Bhardwaj P, Sabapathy SR. Assessment of the hand in cerebral palsy. Indian J Plast Surg 2011;44(2):348-356

18 Colantoni Woodside J, Bindra RR. Rerouting extensor pollicis longus tendon transfer. J Hand Surg Am 2015;40(4):822-825 